\title{
Research on Fault Diagnosis Based on SVM
}

\author{
Li Wanling ${ }^{1, a}$, Wang Zhensheng, Deng Daquan, Tang Jun ${ }^{2}$ \\ ${ }^{1}$ Ordnance Technology Research Institute Shijiazhuang, China \\ ${ }^{2}$ Equipment Department Troop 95829 Xiaogan, China \\ azyh8282180@sina.com
}

Keywords: SVM; fault diagnosis; QPSO; improved QPSO; multi-class SVM

\begin{abstract}
In order to improve the fault diagnosis precision of electron system, a method based on wavelet packet transform and SVM was proposed. Fault diagnosis method based on SVM was researched on in this paper because of the complexity of electron system, difficulty of fault diagnosis method and special advantages of SVM. Wavelet packet transform is used to extract fault features from the signal of the circuit output voltage. The specific feature extraction method is introduced. Improved QPSO algorithm was proposed to improve the training speed and class precision of SVM. At last the method mentioned above was applied to a circuit. The result showed that this method was very good.
\end{abstract}

\section{Introduction}

Electron system is very complex, and connection of subsystems and modules is anfractuous, so the form of fault is very complex, and fault diagnosis for electron system is very difficult.

Fault diagnosis technology is researched on widely and deeply, and its methods are method based on signal process, method based on analytical model and intelligent method based on knowledge.

Fault diagnosis method based on Support Vector Machine (SVM) is an intelligent diagnosis method based on knowledge, SVM establishes a suit of preferable theoretical framework and universal method for machine studying under finite sample, and it has special advantages on solving problems such as little sample, non-linearity, high dimension and local minimum etc, so fault diagnosis method based on SVM was researched on in this paper.

At the same time, improved QPSO algorithm was adopted to train SVM in this paper in order to improve its training speed and diagnosis precision.

\section{Support Vector Machine}

Support Vector Machine (SVM) is a new machine studying algorithm put forward by Vapnik at last century 90 decade metaphase based on Statistical Learning Theory (SLT), and it is used to solve pattern recognition problem firstly. It is based on minimum framework risk principle, and it has very strong generalization capability and can commendably conquer many tradition arithmetic problems such as local minimum, dimension disaster and over-fit etc. And it attracts more and more scholars’ attention.

The main thinking of SVM is using appropriate kernel function to transform input space (nonlinear separable) to a higher dimensional character space (linear separable), and then finding optimal linear hyper plane in the new place. The form of the sorting function is similar to a neural network, its output is linear combination many interface layer nodes, and each interface layer node is inner product of input sample and a support vector. So SVM is also called support vector network.

\section{SVM Effect Factor Analysis}

Factors that affect the performance of SVM include kernel function and its parameters and penalty coefficient C. 
Kernel Function and Its Parameters. Kernel functions commonly used in SVM are linear kernel function, polynomial kernel function, RBF kernel function and sigmoid kernel function etc. Linear kernel function is a special case of RBF kernel function that can map the sample to higher dimension space non-linearly. Polynomial kernel function has more parameters than RBF kernel function that only has one parameter. Polynomial kernel function compares with RBF kernel function is more complex for choosing its model and computing when its frequency is very high. And sigmoid kernel function has wrong instance when some parameters are chosen. So usually RBF kernel function is chosen for SVM.

Penalty Coefficient C. The effect of penalty coefficient $C$ is doing tradeoff between configuration risk and sample error and makes the model has preferable extension capability. If $\mathrm{C}$ is too small, penalty of misjudged samples in the sample data is small, the training error is big, the extension capability of system will change badly, and it will appear lack learning phenomenon. If $\mathrm{C}$ is too big, the learning precision will increase, and the model approaches the actual experience risk minimum, but generalization capability of model will change badly, and it will appear over learning phenomenon. In addition, the value $C$ influences dealing with isolated point in the sample which is selected seemly will have anti-jamming capability at a certain extent to ensure stability for the model.

Data Simulation. In order to validate performance of SVM influenced by its parameters, data of Wine, Breast-cancer, Iris, Hepatitis and Hypothyroid in the UCI database were chosen. Attributes of data was shown as table 1 .

Table 1 UCI Simulation Data

\begin{tabular}{|l|l|l|l|}
\hline Data & Classes & Attribute & Length \\
\hline Wine & 3 & 13 & 178 \\
\hline Breast-cance & 2 & 11 & 286 \\
\hline Iris & 3 & 5 & 153 \\
\hline Hepatitis & 2 & 19 & 155 \\
\hline Hypothyroid & 4 & 29 & 280 \\
\hline
\end{tabular}

Linear, Poly, RBF and Sigmoid kernel functions were adopted to do data simulation, and the results were shown as table 2.

Table 2 Simulation Results of SVM with Different Kernel

\begin{tabular}{|l|l|l|l|l|}
\hline Data & Linea & Poly & RBF & Sigmoi \\
\hline Wine & 0.8989 & 0.904 & 0.927 & 0.7865 \\
\hline Breast-cance & 0.9371 & 0.930 & 0.940 & 0.8042 \\
\hline Iris & 0.8497 & 0.862 & 0.928 & 0.7843 \\
\hline Hepatitis & 0.8774 & 0.877 & 0.929 & 0.7097 \\
\hline Hypothyroid & 0.9036 & 0.928 & 0.928 & 0.8500 \\
\hline
\end{tabular}

From the results, class precision of SVM could be influenced by kernel functions, and class precision of SVM choosing RBF kernel function is higher than others.

\section{Optimization of SVM parameters}

The Improvement of QPSO. Jun Sun, Wenbo Xu and others put forward a new PSO algorithm model in a Quantum Physics’ view [1].

To make a better control of the algorithm's convergence rate, the parameter of contraction and expansion is improved, expansion parameters is as follows.

$$
\alpha(t)=\left\{\begin{array}{l}
\alpha_{\max }-\frac{\alpha_{\text {max }}-\alpha_{\text {min }}}{u \cdot\left(t_{\max }\right)^{3}} \cdot t^{3} \quad, t \leq u \cdot t_{\max } \\
\alpha_{\text {min }}+\frac{\alpha_{\text {max }}-\alpha_{\min }}{(1-u) \cdot\left(t_{\max }\right)^{3}} \cdot\left(t_{\max }-t\right)^{3}, t>u \cdot t_{\max }
\end{array}\right.
$$

There, $u$ is a positive number bellow 1 , the different curves of $u$ are presented in figure 1 . 


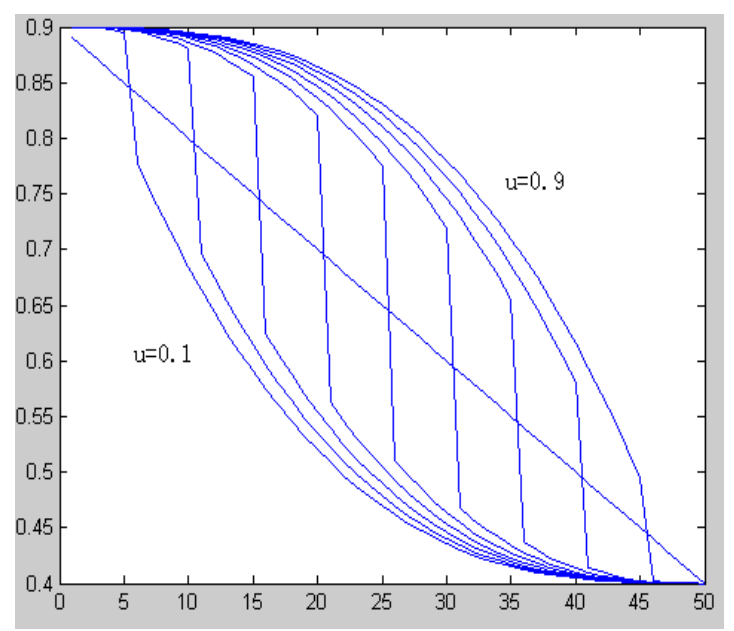

Fig. 1 Different Curves for $u$

As presented in figure 2, the bigger $u$ is better for global search and the smaller $u$ is better for local search, so $u$ is designed to decrease as the increase of iteration times.

Optimization Process. The optimization steps of IQPSO algorithm was shown in the literature [6].

\section{An Example}

$500 \mathrm{~Hz}$ signal generator circuit was chosen to research the diagnosis precision of SVM, and the circuit was shown as figure 2 .

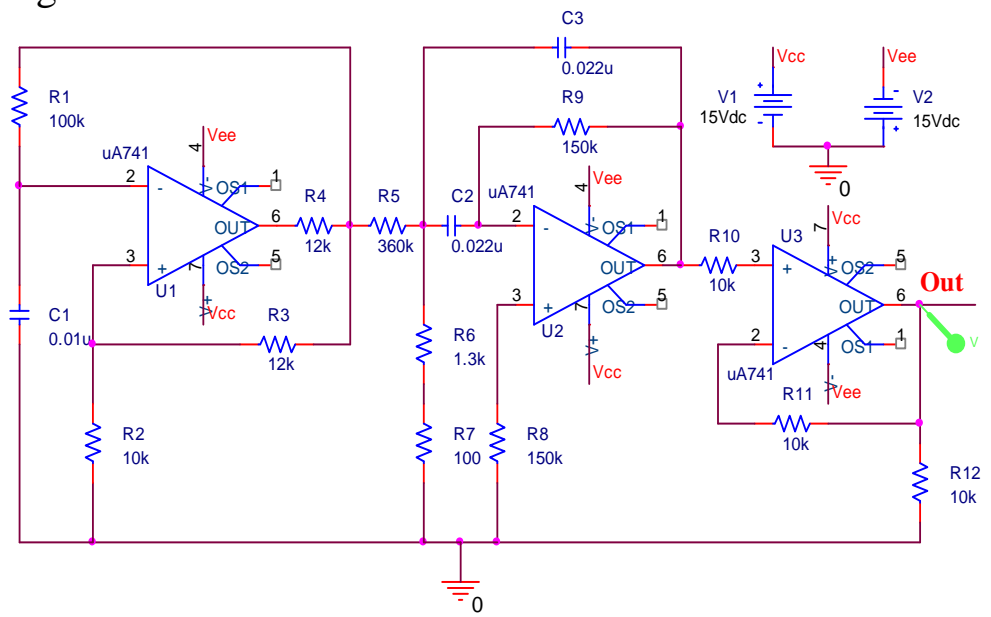

Fig. 2 500Hz Signal Generator

Samples could be got by using Pspice simulation software to simulate circuit 200 Monte-Carlo simulation, joining Signal-to-Noise $10 \mathrm{db}$ gauss white noise in output data, and using Daubechies wavelet db1 wavelet function to do 4 layers wavelet packet disassembling to distill signal character from low frequency to high frequency 16 frequency channels in the 4th layer. Fault states were set for the circuit which was shown as table 3. Necessary samples could be got by doing 200 Monte-Carlo simulation for each state and distilling wavelet packet character.

Table 3 Faults Setting Mode of Analog Circuit

\begin{tabular}{|l|l|l|l|l|}
\hline Parts & \multicolumn{4}{|c|}{ Fault mode } \\
\hline R1 & Fault 1 & $50 \%$ rise & Fault 2 & $50 \%$ descend \\
\hline R3 & Fault 3 & $50 \%$ rise & Fault 4 & $50 \%$ descend \\
\hline R9 & Fault 5 & $50 \%$ rise & Fault 6 & $50 \%$ descend \\
\hline C1 & Fault 7 & 50\% rise & Fault 8 & 50\% descend \\
\hline
\end{tabular}




\begin{tabular}{|l|l|l|l|l|}
\hline C2 & Fault 9 & $50 \%$ rise & Fault 10 & $50 \%$ descend \\
\hline
\end{tabular}

Parameters were optimized by IQPSO algorithm, 100 samples were used to train multi-class SVM, and 100 samples were used as test samples to validate it. The results were shown as table 4.

Table 4 Fault Diagnosis Result of Circuit by Multi-class SVM

\begin{tabular}{|c|c|c|c|c|c|c|c|c|c|c|c|c|}
\hline C & $\gamma$ & \multicolumn{11}{|c|}{ Accuracy matrix } \\
\hline \multirow{11}{*}{76.3087} & \multirow{11}{*}{18.7035} & $\lceil 100$ & 0 & 0 & 0 & 0 & 0 & 0 & 0 & & 0 & \\
\hline & & 0 & 99 & 0 & 0 & 0 & 0 & 1 & 0 & 0 & 0 & 0 \\
\hline & & 0 & 1 & 97 & 0 & 1 & 1 & 0 & 0 & 0 & 0 & 0 \\
\hline & & 1 & 0 & 1 & 8 & 0 & 0 & 0 & 0 & 0 & 0 & 0 \\
\hline & & 0 & 1 & 1 & 0 & 95 & 2 & 0 & 0 & 1 & 0 & 0 \\
\hline & & 0 & 0 & 0 & 0 & 1 & 97 & 1 & 0 & 0 & 0 & 1 \\
\hline & & 0 & 0 & 2 & 1 & 0 & 0 & 94 & 1 & 0 & 2 & 0 \\
\hline & & 0 & 0 & 0 & 0 & 0 & 2 & 1 & 92 & 3 & 1 & 1 \\
\hline & & 1 & 1 & 0 & 0 & 2 & 0 & 3 & 0 & 93 & 0 & 0 \\
\hline & & 0 & 1 & 1 & 2 & 0 & 0 & 0 & 0 & 0 & 96 & 0 \\
\hline & & 0 & 0 & 1 & 1 & 0 & 1 & 0 & 0 & 0 & 2 & 95] \\
\hline
\end{tabular}

From above, using RBF kernel function and adopting IQPSO algorithm to optimize kernel function parameters and penalty coefficient $\mathrm{C}$ could improve the class precision of SVM and fault diagnosis accuracy.

\section{Conclusions}

Intelligent diagnosis method based on SVM was adopted because of the complexity of electron system, difficulty of fault diagnosis method and special advantages of SVM, and the effect factors were analyzed. From simulation analysis, class precision of RBF kernel function was very high. Improved QPSO arithmetic was proposed to optimize parameters of SVM to improve its train speed and class precision. A fault diagnosis method based on wavelet packet transform and SVM was proposed and was applied to a circuit. Fault diagnosis of circuit was realized. Wavelet function was used to do 4 layers wavelet packet disassembling to distill energy of each frequency channel in the 4th layer as fault characters. The result showed that diagnosis speed and precision of the method were very high, and it has wide application foreground.

\section{References}

[1] Jun Sun, Wenbo Xu. A Global Search Strategy of Quantum-behaved Particle Swarm Optimizations [C]. IEEE 2004 Conference on Cybernetics and Intelligent System, Singapore: $111 \sim 116$.

[2] Wang Cheng. Study on Fault Diagnosis in Analog Circuits Based on Neural Networks[D].Sichuan: University of Electronic Science and Technology of China.

[3] Vapnik V. The Nature of Statistical Learning Theory[M]. New York: Springer-Verlag, 1999.

[4] C. W. HSU. A Comparison of Methods for Multiclass Support Vector Machines[C]. IEEE Transactions on Neural Networks, Vol. 13, No. 2, pp. 415-425, 2002.

[5] Tian Yingjie, Qi Zhiquan. A New Support Vector Machines for Multi-class Classfication [C] // Proceedings of 2nd International Conference on Recent Advances in Space Technologies. 2005: 18-22.

[6] Li Wanling, Han Lei, Song Xiangjun. Research on Prognostic Method Based on BP Optimized by Improved QPSO [C] // International Conference on Electric Technology and Civil Engineering. 2012: 2625-2628. 